Case

Report

\title{
Pulmonary Myofibroblastic Tumour Involving the Pericardium and Left Atrium in an 18 Month Infant
}

\author{
Ruben Lamas-Pinheiro, MD, ${ }^{1,2}$ Gregory Rodesch, MD, ${ }^{2}$ Christine Devalck, MD, ${ }^{3}$ \\ Valérie Segers, MD,${ }^{4}$ Karim Khelif, MD,${ }^{2}$ Matteo Cappello, MD,${ }^{5}$ and Henri Steyaert, MD ${ }^{2}$
}

\begin{abstract}
Inflammatory myofibroblastic tumor (IMT) is the most frequent primary lung tumor in children and it may be locally aggressive. The management of a locally advanced pulmonary IMT in an 18 month-old female child is presented.

A left pulmonary mass was incidentally found on the computerized tomography (CT) scan of a child with persistent systemic inflammatory syndrome. Biopsy confirmed the diagnosis; after preoperative corticotherapy, left pneumonectomy was performed. The pericardium and left atrium were invaded and resected, requiring pericardial reconstruction. There is no relapse at four years of follow-up.

Steroids play a role in tumor size reduction, but marginal resection is the gold standard. Extended approaches are feasible and often required in advanced cases.
\end{abstract}

Keywords: myofibroblastic tumour, atrium, pneumonectomy, lung, pericardium

\section{Introduction}

Inflammatory myofibroblastic tumour (IMT) accounts for $1 \%$ of pulmonary neoplasms, with an incidence of $0.04 \% .{ }^{1)}$ IMT occurs at all ages, but more than $35 \%$ of cases occur in children. ${ }^{1)}$ Although initially considered benign, the question remains if it is a reactive pseudotumorous

${ }^{1}$ Departmant of Pediatric Surgery, Hospital São João, Porto, Portugal

${ }^{2}$ Department of Pediatric Surgery, University Children Hospital Queen Fabiola, Free University of Brussels, Belgium

${ }^{3}$ Hematology/Oncology Unit, University Children Hospital Queen Fabiola, Free University of Brussels, Belgium

${ }^{4}$ Department of Anatomopathology, University Hospital Brugmann, Brussels, Belgium

${ }^{5}$ Department of Thoracic Surgery, Erasme Hospital, Free University of Brussels, Belgium

Received: December 15, 2015; Accepted: January 12, 2016 Corresponding author: Ruben Lamas-Pinheiro, MD. Pediatric Surgery Department, Hospital São João, Alameda Prof. Hernâni Monteiro, 4200-319 Porto, Portugal

Email: rubenlms@hotmail.com

(C)2016 The Editorial Committee of Annals of Thoracic and Cardiovascular Surgery. All rights reserved. proliferation or a true neoplasm, as some authors consider it as an inappropriate immunologic response. ${ }^{1)}$ The World Health Organization (WHO) recognized IMT as a lowgrade mesenchymal malignancy with a local recurrence rate of $10 \%-20 \%$ and a low risk of distance metastases. ${ }^{2)}$ Anaplastic lymphoma kinase (ALK) gene mutations have been found in a significant fraction of IMT corroborating its potential malignant nature. ${ }^{1)}$ The treatment of choice for pulmonary IMT is complete resection. ${ }^{3,4)}$ Chemotherapy, radiation, and anti-inflammatory drugs have been proposed as adjuvant or alternative therapies. $\left.{ }^{4}\right)$

The authors describe a pulmonary IMT invading the pericardium and left atrium, requiring left pneumonectomy, partial atrial resection and pericardial reconstruction.

\section{Case}

A large left upper lobe mass was incidentally found on computerized tomography (CT) scan during work-up of a pulmonary infection in a previously healthy 18 month-old girl (Fig. 1). The child was treated with antibiotics and a lung biopsy was performed. Histologic examination 


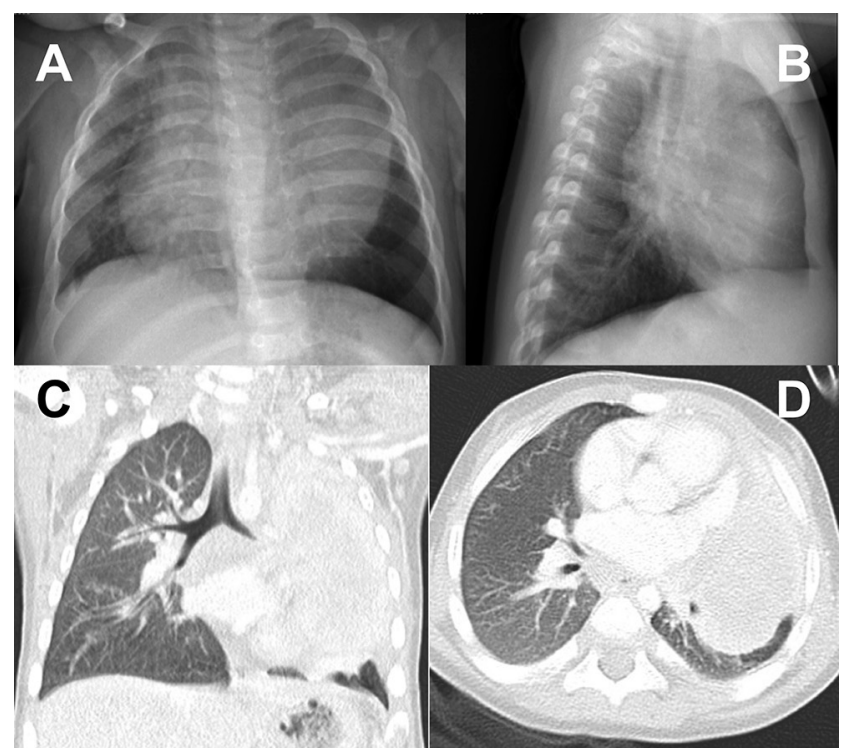

Fig. 1 Chest roentgenogram (A, B). Thoracic computerized tomography scan $(\mathbf{C}, \mathbf{D})$ : Left upper lobe tumor crossed by the left pulmonary vessels, separated from the thymus and without anomalous feeding vessels.

revealed pneumonia with follicular bronchiolitis. Two months after, there was no tumor size reduction and a systemic inflammatory syndrome persisted: hypochromic microcytic anemia (hemoglobin $8.4 \mathrm{~g} / \mathrm{dL}$ ), elevated erythrocyte sedimentation rate $(120 \mathrm{~mm} / \mathrm{h})$, increased C-reactive protein levels $(10.2 \mathrm{mg} / \mathrm{dL})$ and elevated thrombocyte count $(764000 / \mu \mathrm{L})$. Lung biopsy was repeated confirming the diagnosis of ALK negative IMT (Fig. 2).

Steroids were initiated (one month prednisolone, $0.2 \mathrm{mg} / \mathrm{kg} /$ day), followed by surgical resection. A left side thoracotomy, through the fifth intercostal space, was performed. Adhesions were divided, the tumor mobilized and a wider invasion identified: extending along the fissure to the central portion of the inferior lobe, including the left main bronchus. Left pneumonectomy was needed in the presence of bi-lobar involvement (Fig. 2). The pericardium was invaded and resected $(6 \mathrm{~cm} \times 7 \mathrm{~cm})$ sparing the phrenic nerve. The left atrium was also involved and partial atrial resection was needed. Pericardial reconstruction was performed using Parietex ${ }^{\circledR}$. A chest tube was introduced and removed after 3 days.

Postoperative period was uneventful, the child being discharged from intensive care unit on the third post-operative day and left hospital on the seventh. At three years of follow-up, the child is in good over-all condition, gaining weight, without scoliosis or postpneumonectomy syndrome and no clinical or radiological relapse.

\section{Discussion}

Patients with pulmonary IMT can be asymptomatic or present with recurrent pulmonary infections, cough, chest pain, hemoptysis and dyspnea. Systemic symptoms and laboratory evidence of systemic inflammatory response is present in $30 \%-50 \%$ of patients. As the tumor produces interleukins these findings frequently resolve after surgery and reappearance may indicate a possible recurrence. ${ }^{1)}$

Chest roentgenogram may reveal a suspicious radiopaque image, CT scan features are unspecific and there is no role for magnetic resonance image in the initial investigation. ${ }^{2)}$ Metastatic/multifocal lesions in the central nervous system have been reported and screening has been suggested at diagnosis and/or follow-up. ${ }^{5)}$ Radiological findings may raise suspicion, but are not reliable for diagnosis.

A proper biopsy is challenging to obtain: central lesions require bronchoscopy and peripheral lesions thoracoscopy or image-guidance. Gross appearance is firm or gelatinous with white or tan cut surface (Fig. 2A) ${ }^{2)}$ Histology is characterized by spindle cells, inflammatory infiltrate with plasma cells and lymphocytes in a myxoid to collagenous stroma. Three histological patterns were described (myxoid, compact spindle cell and fibrous hypocellular), they may coexist and none defines aggressiveness. ${ }^{1)}$ IMT expresses smooth muscle actin, vimentin, desmin and calponin, but there is no specific marker. ${ }^{2)}$ Therefore molecular biology or fluorescence in situ hybridization (FISH) must rule out a sarcoma. ${ }^{2}$

Chromosomal abnormalities were described, including rearrangements of ALK locus on chromosome 2p23. ${ }^{2}$ In immunohistochemistry or by FISH near 50\% are ALKpositive, more frequently in younger patients, but no prognostic association was established.2)

Complete resection is the gold standard treatment. ${ }^{1-3)}$ Wedge resection may be appropriate for small peripheral lesions and lobectomy can be reserved for central larger tumors. En bloc resections are required in case of invasion of the chest wall, cervico-thoracic junction, mediastinum or diaphragm to ensure favorable long-term results. ${ }^{3)}$ An association between the mediastinal extension and increased disease progression, recurrence and mortality has been suggested; bilobar involvement may require pneumonectomy; this procedure is rarely carried out during childhood and data regarding the outcome and prognosis is limited.2) Reported complications include reduction of pulmonary function, failing to thrive, chest, 


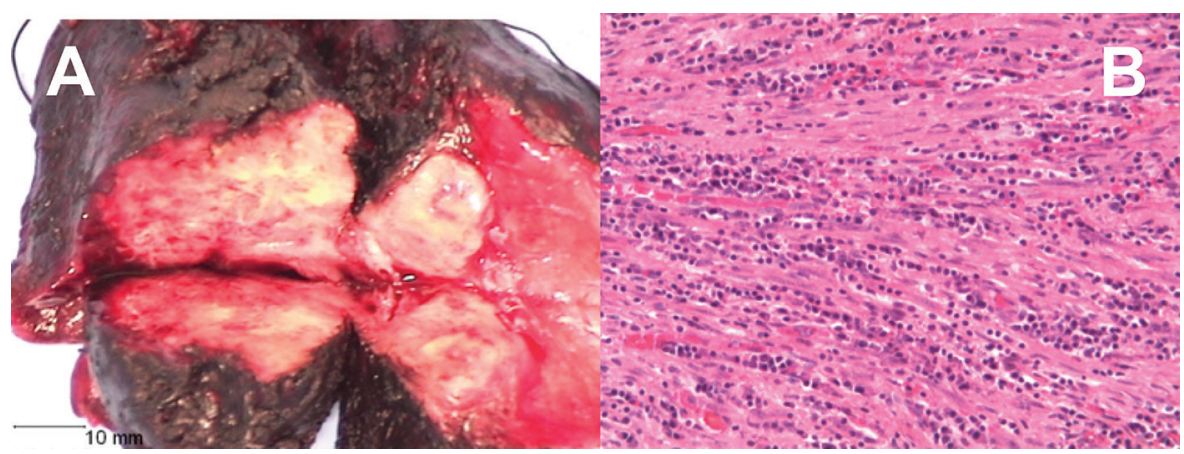

Fig. 2 Gross appearance of the resected mass (A). Histology findings (B): fusiform cells and inflammatory infiltrate with plasma cells and lymphocytes; positive stain for $\alpha$-smooth muscle actin, with no ALK overexpression. ALK: anaplastic lymphoma kinase

spinal deformities and the rare postpneumonectomy syndrome with dynamic airway obstruction caused by extreme rotation and shift of the mediastinum, resulting in symptomatic central airway compression.

Radiation is the last option with debatable efficacy and significant morbidity. ${ }^{3)}$ Steroids play an important role in pre-operative tumor size reduction and post-operative relapse. ${ }^{3)}$ Other chemotherapeutics agents were used, but the variety of cells involved in this lesion makes it difficult to elect an agent. Crizotinib (ALK-inhibitor) is part of an ALK-directed therapy emerging as highly effective option for ALK-positive cases.

WHO has recently reclassified IMT as low-grade malignancy. ${ }^{2)}$ Several studies demonstrated infrequent recurrence after complete resection; however, metastatic disease is identified either at presentation or within first year of diagnosis and some patients develop it several years after surgery. $\left.{ }^{4}\right)$ Regular follow-up seams mandatory, but no protocol has been proposed.

Physicians should be aware of this entity and its challenging medical and surgical management. Particularly in children, these lesions are more invasive. Extended surgical approach in mediastinic invasion is feasible and often required in complex cases.

\section{Disclosure Statement}

The authors have no conflict of interest.

\section{References}

1) Coffin CM, Hornick JL, Fletcher CD. Inflammatory myofibroblastic tumor: comparison of clinicopathologic, histologic, and immunohistochemical features including ALK expression in atypical and aggressive cases. Am J Surg Pathol 2007; 31: 509-20.

2) Marie-Cardine A, Berrebi D, Orbach D. Guidelines for management of localized inflammatory myofibroblastic tumours in children. Bull Cancer 2011; 98: 209-16.

3) Fabre D, Fadel E, Singhal S, et al. Complete resection of pulmonary inflammatory pseudotumors has excellent long-term prognosis. J Thorac Cardiovasc Surg 2009; 137: 435-40.

4) Morotti RA, Legman MD, Kerkar N, et al. Pediatric inflammatory myofibroblastic tumor with late metastasis to the lung: case report and review of the literature. Pediatr Dev Pathol 2005; 8: 224-9.

5) Siminovich M, Galluzzo L, López J, et al. Inflammatory myofibroblastic tumor of the lung in children: anaplastic lymphoma kinase (ALK) expression and clinico-pathological correlation. Pediatr Dev Pathol 2012; 15: 179-86. 